\title{
Enhanced Self-Assembly of Pyridine-Capped CdSe Nanocrystals on Individual Single-Walled Carbon Nanotubes
}

\author{
Qingwen Li $i^{a}$, Baoquan Sun ${ }^{b}$, Ian A. Kinloch ${ }^{a}$, Dan Zhi ${ }^{a}$, Henning Sirringhaus ${ }^{b}$ \\ Alan H. Windle
}

\section{Supporting Materials}
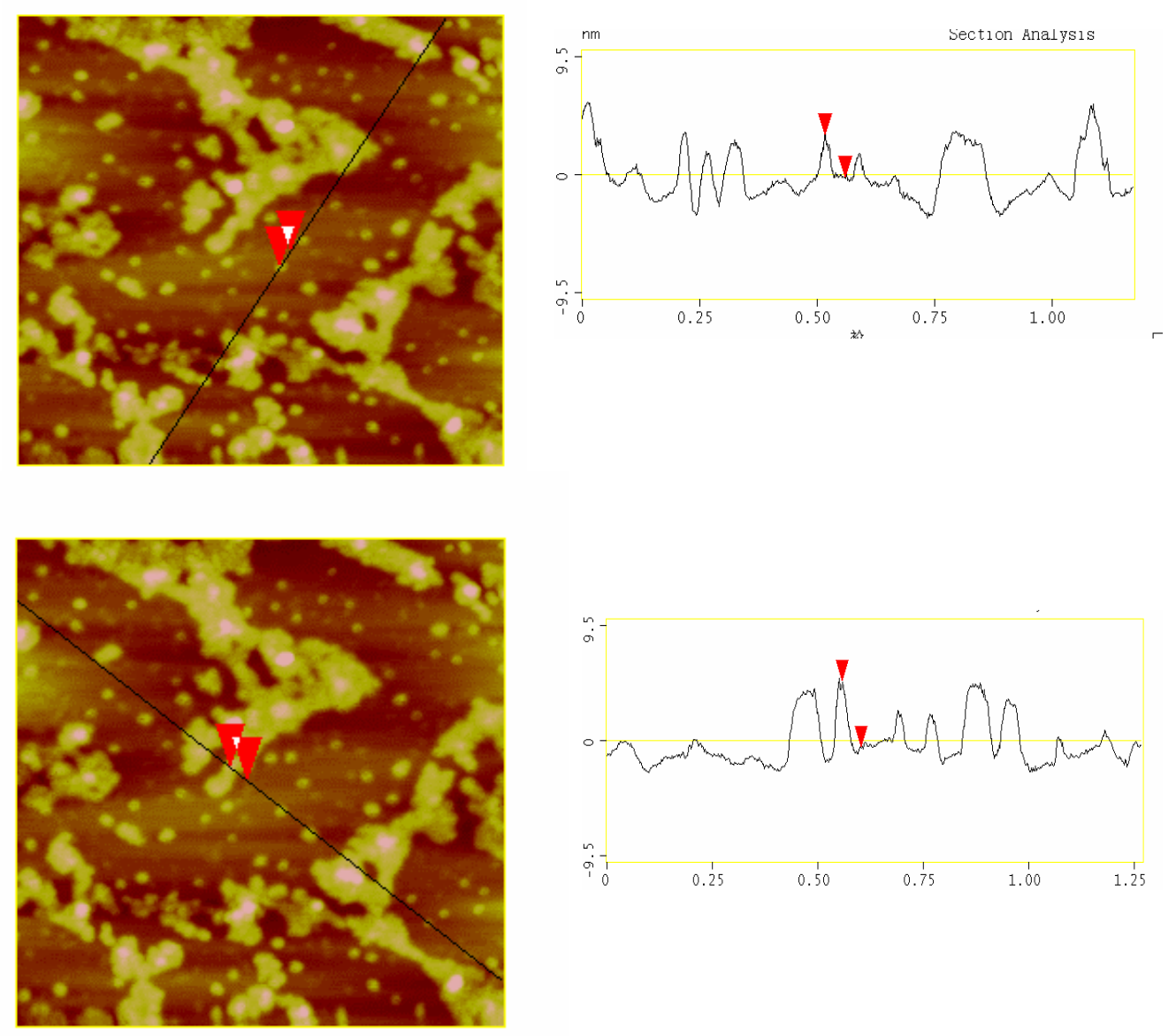

Figure 1. AFM images and cross-section analysis of TOPO capped QDs absorbed onto HOPG surface. Top: Discrete nanoparticles; Bottom: A large feature 


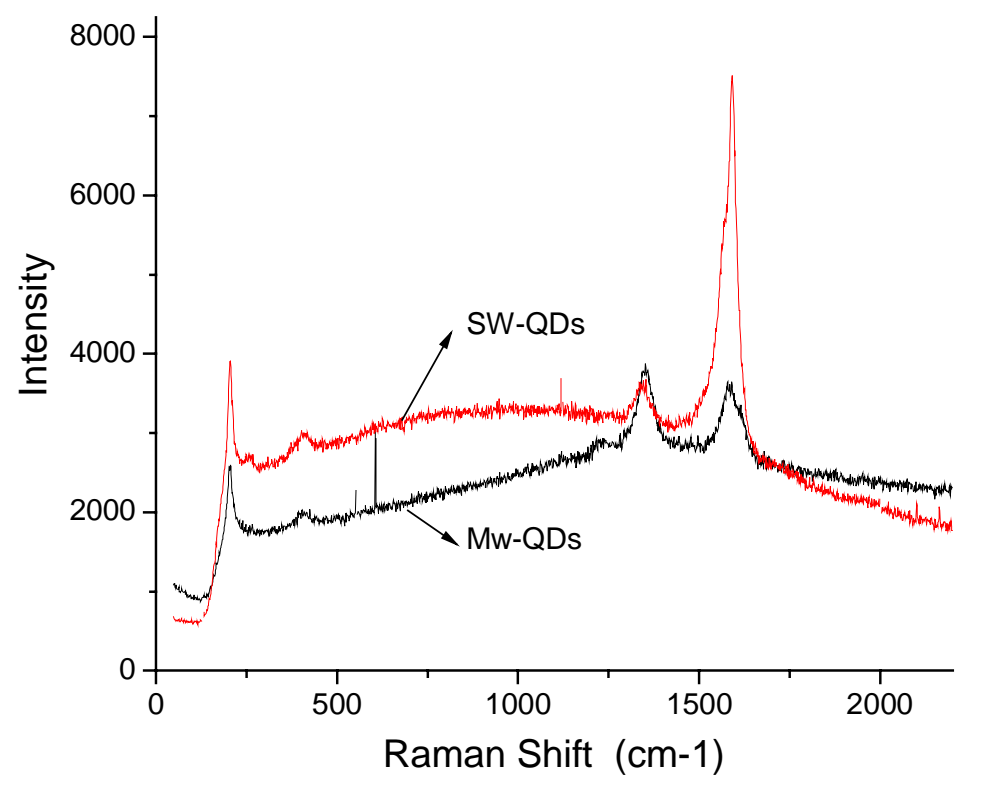

Figure 2. Comparative studies on the Raman spectra of carbon nanotube and Py-QDs conjugates indicating that the peaks at low wavenumbers came from the contribution of QDs. 